\title{
Avant-propos
}

\section{Une revue pour des agricultures en mouvement}

$\mathrm{D}$ epuis sa création, il y a 20 ans, sous l'impulsion de l'Agence universitaire de la Francophonie (AUF), la revue Cahiers Agricultures a permis à de nombreux auteurs (chercheurs, enseignants et professionnels des agricultures du Nord comme du Sud) de faire connaître à un lectorat varié leurs travaux, leurs réflexions ou leurs opinions sur les problèmes que rencontrent les sociétés rurales et les innovations susceptibles de les résoudre.

La diversité des sujets abordés dans la revue constitue à la fois un atout et un handicap pour une revue scientifique qui souhaite publier des textes originaux et scientifiquement fondés mais aussi socialement pertinents. Cette diversité de sujets est un atout quand elle permet de refléter la diversité des "agricultures ", sensu lato, au travers de leurs processus de production, de transformation, de qualification et de mise en marché. Chaque fois qu'il est possible de le faire, l'approche comparée des pratiques agricoles et des filières de production permet d'ailleurs, dans des synthèses, de donner du sens à des processus géographiquement et socialement situés, pour des lecteurs non spécialistes.

Cependant, cette diversité constitue aussi un handicap, car elle peut rebuter des auteurs cherchant à atteindre des lectorats de spécialistes, dans un souci de faire connaître et reconnaître l'excellence de leurs idées, et si possible de les voir citer en référence dans la littérature mondiale.

La tension perceptible dans les systèmes d'évaluation des recherches entre excellence scientifique et pertinence sociale, entre reconnaissance par les pairs et réponse à la demande sociale, se retrouve donc dans notre revue, mais quoi de plus normal. Cette tension s'illustre en effet dans les propos d'Ester Duflo, lors de sa leçon inaugurale au Collège de France ${ }^{1}$, lorsqu'elle déclare, par exemple: "Les grandes figures du débat public sur la pauvreté et le développement ne publient plus leurs travaux dans les revues scientifiques: ils ne survivraient pas aux comités de lecture. "

Cette constatation dérangeante suit par ailleurs de peu la publication du livre Agronomes et paysans ${ }^{2}$, d'André Pochon, agriculteur breton à l'origine de multiples innovations qui ont fait progresser l'élevage bovin dans sa région. Il y décrit ses 50 années de dialogue musclé avec les chercheurs de l'Inra, faites de périodes de collaboration, certes, mais aussi d'affrontements violents.

Le comité de rédaction des Cabiers Agricultures se trouve ainsi confronté à un réel défi: faire vivre une revue que nous voulons ouvrir aux problèmes du développement et de la lutte contre la pauvreté, tout en permettant aux chercheurs, du Sud comme du Nord, de s'exprimer et de se faire reconnaître par leurs pairs.

La revue Cahiers Agricultures, avec sa nouvelle équipe de rédacteurs en chef, poursuivra donc, en 2009, ses efforts pour susciter des articles de fond sur des questions de société qui engagent l'avenir, mais qui, suscitant l'émotion plus que la réflexion, ne reçoivent pas toujours de réponses scientifiquement fondées. Telles ont été, ces derniers mois, les questions relatives aux "émeutes de la faim " dans les métropoles de certains pays du tiers-monde, telles seront demain celles que ne vont pas manquer de poser les stratégies d'accaparement des terres cultivables par des États et des sociétés mondialisées, au détriment des agricultures familiales, pour s’affranchir des aléas et contraintes des marchés internationaux.

Au cours de ces dernières années, sous l'impulsion de René Lésel, la revue a progressé, recevant un nombre croissant d'articles à publier, accroissant sa diffusion et réalisant des numéros thématiques qui ont connu un large succès. En 2007, les Cahiers Agricultures ont été référencés par l'ISI et ont obtenu, en 2008, un premier facteur d'impact. Cette avancée notable mérite d'être confortée dans les années à venir, mais elle devra déjà être largement diffusée au sein des institutions qui soutiennent la revue et auprès des auteurs qui sont susceptibles de lui confier des textes de qualité.

La revue souhaite à cet effet s'ouvrir sur les sociétés savantes des pays du Nord et du Sud et sur les réseaux professionnels, parmi lesquels ceux de l'AUF qui développent leurs activités dans le champ couvert par sa ligne éditoriale.

Dans l'avenir, le comité de rédaction continuera parallèlement à encourager les auteurs des pays en développement à lui soumettre leurs articles. Il s'engage, par exemple, à assurer un appui éditorial aux auteurs francophones des institutions d'enseignement supérieur et de recherche des pays en développement qui développent des idées originales. Le comité de rédaction souhaite à cet effet impliquer davantage, dans son fonctionnement, des membres des pays du Sud, par exemple, en utilisant davantage les ressources qu'offre Internet.

Espérons qu'ainsi les Cahiers Agricultures donneront aux lecteurs une meilleure image de la diversité et de la complexité des agricultures du monde.

Didier Picard

$<$ Didier.Picard@grignon.inra.fr $>$ Jean-Pascal Pichot <jeapichot@numericable.fr>

$\overline{1 \text { Le Monde, }} 20$ janvier 2009.

2 Pochon A. Agronomes et paysans. Collection "Sciences en question ". Versailles: éditions Quae, 2008. 